\title{
Cuidados Paliativos: Prática dos Médicos da Estratégia Saúde da Família
}

\section{Palliative Care: Practice of Family Health Strategy Physicians}

Júlia Rezende Ribeiro ${ }^{I}$ Kátia Poles ${ }^{1}$

\section{PALAVRAS-CHAVE}

- Cuidados Paliativos.

- Medicina Paliativa.

- Atenção Primária à Saúde.

- Educação Médica.
Com o avanço do conhecimento científico e aumento da expectativa de vida, criou-se a ilusão de que o ser humano é imortal. Ademais, existe uma deficiência na educação médica atual, que forma profissionais tecnicistas e sem preparo para cuidar de um paciente com doença grave e incurável. Por volta da metade do século XX, surgiram movimentos voltados para a humanização dos atendimentos em saúde, que levavam em consideração a integralidade do indivíduo. Cuidado paliativo é uma abordagem que melhora a qualidade de vida de pacientes e familiares diante de doenças que ameacem a continuidade da vida, por meio do alívio do sofrimento, tratamento da dor e de outros sintomas de natureza física, psicossocial e espiritual. Considerando que esse tipo de cuidado pode ser oferecido em diferentes contextos, ressalta-se que a Atenção Primária em Saúde é uma possibilidade recente para a assistência em cuidados paliativos. Entretanto, ainda existe uma dificuldade dos serviços de Atenção Primária no Brasil em oferecer essa modalidade de assistência. Assim, a pesquisa teve como objetivo compreender a percepção dos médicos da Estratégia Saúde da Família acerca dos cuidados paliativos. Trata-se de estudo descritivo, com abordagem qualitativa, em que foi utilizada a metodologia da análise temática. Os participantes foram 16 médicos que atuam nas Unidades Estratégia Saúde da Família do município de Lavras, Minas Gerais. Os dados foram coletados por meio de entrevista semiestruturada. Com base na análise dos dados identificou-se que os médicos possuem conhecimento incipiente sobre o conceito de cuidados paliativos e que, embora tenham tido algum tipo de experiência com esse tipo de paciente, apresentam dificuldade para abordá-lo de maneira holística. Isto se deve, principalmente, à deficiência na formação acadêmica, que ainda privilegia o conhecimento biomédico e a cura das doenças em detrimento do alívio do sofrimento humano. Conclui-se que é imprescindível abordar o tema durante a formação médica, bem como discuti-lo entre os profissionais já atuantes nos serviços de saúde para que seja possível proporcionar melhor qualidade de vida e de morte. 


\section{KEY-WORDS}

- Palliative Care.

- Palliative Medicine.

- Primary Health Care.

- Education, Medical.

\begin{abstract}
With the advance of scientific knowledge and increased life expectancy, the illusion has been created that the human being is immortal. In addition, there is a deficiency in current medical training, whereby professional technicians are trained without being prepared to take care of a patient with serious and incurable disease. Towards the middle of the $20^{\text {th }}$ century, there were movements aimed at the humanization of health care, which took into account the integrality of the individual. Palliative care is an approach that improves the quality of life of patients and their families in the face of life-threatening illnesses by relieving suffering, treating pain, and other symptoms of a physical, psychosocial and spiritual nature. Considering that this type of care can be offered in different contexts, it has recently been highlighted that Primary Health Care can be a suitable setting for palliative care assistance. However, there is still a difficulty for Primary Care services in Brazil to offer this kind of assistance. Thus, the research was aimed at understanding the perception of physicians of the Family Health Strategy about palliative care. A descriptive study, with a qualitative approach, using thematic analysis method. The participants were 16 physicians who work in the Family Health Strategy Units of the city of Lavras, Minas Gerais. Data were collected through a semi-structured interview. From the data analysis it was identified that the doctors have incipient knowledge regarding the concept of palliative care and that although they had some type of experience with this type of patient, they encounter difficulties in approaching it in a holistic way. This is mainly due to the deficiency in academic training, which still favors biomedical knowledge and the cure of diseases to the detriment of the relief of human suffering. It is concluded that it is essential that this topic be addressed during medical training, as well as discussed among the professionals already working in the health services so as to provide a better quality of life and death.
\end{abstract}

Recebido em: 22/1/19

Aceito em: 20/2/2019

\section{INTRODUÇÃO}

Com o desenvolvimento da ciência e da tecnologia, dos medicamentos e tratamentos, houve um aumento da expectativa de vida e da cura de doenças antes consideradas letais. Dessa forma, assistiu-se ao envelhecimento da população e ao aumento da incidência de doenças crônico-degenerativas não transmissíveis (DCNT) $)^{1,2}$.

Ao lado desse processo, a medicina adquiriu um aspecto mais tecnicista e biologicista, focando as doenças e não o indivíduo como um todo. Esses fatores contribuíram para a formação de médicos centrados em tratar as desordens orgânicas e não o doente, e que alimentam a falsa ilusão de que conseguem curar todas as doenças, vendo a morte como sinônimo de fracasso ${ }^{3}$.

Em contraponto a esse modelo, na metade do século XX surgiram movimentos voltados à humanização da Medicina $\mathrm{e}$ dos atendimentos em saúde, que levavam em consideração a integralidade do indivíduo, buscando tratá-lo como um todo biopsicossocial. Simultaneamente a esse processo, desenvolveu-se o movimento Hospice Moderno, pelas mãos da médica, enfermeira e humanista inglesa Cicely Saunders, que fundou, em 1967, o St Cristopher's Hospice, instituição voltada à assistência, ao ensino e à pesquisa com pacientes em fim de vida ${ }^{1}$. Nesse momento, surgiram também os trabalhos de Elizabeth Kubler-Ross, médica psiquiatra, autora de diversos livros, entre eles On Death and Dying, onde escreve sobre um assunto até então tabu nas sociedades ocidentais: a morte e o morrer".

Em 1982, o Comitê de Câncer da Organização Mundial da Saúde (OMS) criou um grupo responsável por delimitar políticas que aliviassem a dor e cuidados que pudessem ser recomendados em todos os países para pacientes com câncer. Ainda na década de 1980, o termo cuidados paliativos (CP) passou a ser usado pela OMS, que publicou sua primeira definição de cuidados paliativos em $1990^{1}$. Segundo definição da $\mathrm{OMS}^{5}$, cuidado paliativo é:

Uma abordagem que promove qualidade de vida dos usuários (adultos e crianças) e suas famílias que enfrentam problemas associados a doenças que ameaçam a vida. Previne e alivia o sofrimento através da identificação precoce, avaliação correta e tratamento da dor e outros problemas, físicos, psicossociais ou espirituais. 
No Brasil, os cuidados paliativos começaram a se desenvolver na década de 1980. Em 1997, esses serviços experimentam uma expansão, com a criação da Associação Brasileira de Cuidados Paliativos. Em 1998, o Instituto Nacional do Câncer (Inca) inaugurou, em seu hospital, uma ala voltada somente ao tratamento de pacientes em cuidados paliativos. Em 2005, foi criada a Academia Nacional de Cuidados Paliativos (ANCP), e em 2011 o Conselho Federal de Medicina (CFM) reconheceu os cuidados paliativos como área de atuação médica, a partir da Resolução CFM 1973/20116,7.

A Atenção Primária é o primeiro acesso ao sistema de saúde de seus usuários e objetiva oferecer cuidado integral, coordenando e integrando o atendimento prestado por outros serviços de saúde. A Estratégia Saúde da Família (ESF) é a principal representante da Atenção Primária no Brasil. Foi criada em 1994 a partir do Programa de Agentes Comunitários (PACS), com a intenção de reorganizar a assistência à saúde, em conformidade com os princípios do Sistema Único de Saúde (SUS) $)^{2,8}$.

No Brasil, já foram promulgadas normas que estabelecem o cuidado paliativo e o atendimento domiciliar no SUS. Entre elas, pode-se citar a Portaria n⿳o 19 de janeiro de 2002, que amplia a inserção dos cuidados paliativos no SUS através do Programa Nacional de Assistência à Dor e Cuidados Paliativos, e a Lei no 10.424 , de abril de 2002, que regulamenta o atendimento e a internação domiciliar dentro do SUS. Mais recentemente, desenvolveu-se também o Programa Melhor em Casa, que visa fortalecer o atendimento domiciliar pelas equipes de Atenção Primária. Entretanto, ainda são poucos os locais em que os cuidados paliativos são praticados pelas equipes de ESF${ }^{8-12}$.

Em muitos países, porém, a Atenção Primária em Saúde (APS) é classificada como o melhor nível de assistência à saúde para prestação de serviços de cuidados paliativos. Entende-se que a proximidade emocional, cultural e geográfica dos profissionais contribui para que o cuidado seja humanizado, respeitando-se a autonomia do paciente e de suas famílias e evitando-se a fragmentação do indivíduo. Além disso, a APS permite que o indivíduo permaneça em casa, de forma a evitar seu afastamento da família nos momentos finais de vida ${ }^{8,12}$.

Um estudo brasileiro ${ }^{3}$ que teve por objetivo conhecer o estado atual da prática dos cuidados paliativos evidenciou que a maioria dos médicos ligou essa área apenas ao alívio do sofrimento físico e qualidade de vida, esquecendo-se dos aspectos sociais, psicológicos e espirituais, além de não enquadrar a família do paciente dentro da abordagem de um serviço de cuidados paliativos. De acordo com outro estudo ${ }^{12}$, diversos motivos dificultam a incorporação dos cuidados paliativos na Atenção Primária em Saúde, entre eles a falta de recursos tecnológicos e a dificuldade dos profissionais em lidar com as famílias. Essa dificuldade pode ter origem na não obrigatoriedade da abordagem do tema cuidados paliativos nas escolas de ensino médico brasileiras ${ }^{13}$. Conforme estudo de Figueiredo e Stano ${ }^{14}$, em Minas Gerais, apenas a Faculdade de Medicina de Itajubá oferecia, em 2013, a disciplina de Tanatologia e Cuidados Paliativos no curso de Medicina. Essa deficiência se perpetua após o término da graduação, visto que são poucos os cursos de especialização e residência em Medicina Paliativa no Brasil.

É visível, portanto, que os cuidados paliativos ainda constituem uma área pouco trabalhada e explorada na medicina, apesar dos inúmeros avanços experimentados.

\section{OBJETIVOS}

\section{Objetivo geral}

- Compreender a percepção de médicos da Estratégia Saúde da Família com relação aos cuidados paliativos.

\section{Objetivos específicos}

- Identificar as práticas de Cuidados Paliativos por médicos da Estratégia Saúde da Família;

- Identificar as dificuldades encontradas pelos médicos da Estratégia Saúde da Família quanto ao atendimento de pacientes em Cuidados Paliativos.

\section{METODOLOGIA}

Este é um estudo descritivo, com abordagem qualitativa, que se realizou com 16 médicos que trabalham nas Unidades Estratégia Saúde da Família em um município do Sul de Minas Gerais. Esse tipo de pesquisa se direciona a interpretar a experiência humana, tal como é definida e vivida pelos sujeitos e suas percepções ${ }^{15}$.

No presente estudo, foi utilizada a metodologia da análise temática. Esta é uma técnica que, por meio da leitura e interpretação do conteúdo das entrevistas, permite a realização de análises que conduzem a descrições objetivas, sistemáticas e qualitativas das comunicações, auxiliando a reinterpretá-las e a atingir uma compreensão aprofundada de seus significados ${ }^{16}$.

Os dados foram coletados por meio de entrevistas com roteiro semiestruturado em local privado nas Unidades Estratégia Saúde da Família no referido município.

O projeto foi aprovado pelo Comitê de Ética em Pesquisa com Seres Humanos da Universidade Federal de Lavras (Ufla) (CAAE: 59408116.1.0000.5148), atendendo à Resolução 466/2012 do Conselho Nacional de Saúde do Ministério da Saúde, que dispõe sobre diretrizes e normas regulamentares da pesquisa envolvendo seres humanos ${ }^{17}$. 


\section{RESULTADOS}

O município em que a pesquisa foi desenvolvida conta com 17 Unidades Estratégia Saúde da Família. Dezesseis médicos foram entrevistados e voluntariamente aceitaram participar da pesquisa; uma médica não consentiu em participar do estudo. A caracterização dos entrevistados está apresentada no Quadro 1.

Foram entrevistados oito profissionais do sexo masculino e oito do sexo feminino. As idades variaram entre 25 e 67 anos. O tempo de formação variou entre um e 38 anos. Dos entrevistados, sete não possuíam algum tipo de especialização ou residência médica, enquanto os outros nove possuíam especialização ou residência. Nenhum dos entrevistados tinha especialização ou residência em Medicina de Família e Comunidade, evidenciando uma lacuna na formação para atuação na Atenção Primária à Saúde. Um dos entrevistados era de nacionalidade cubana e trabalhava como Médico de Saúde da Família no Programa Mais Médicos do governo federal.

Com base na análise do discurso, identificaram-se os seguintes temas: conceituação de cuidados paliativos, formação acadêmica, experiências de atendimento, sentimentos relacionados à morte, limitações dos profissionais e do sistema de saúde, comentados a seguir.

\section{Conceituação de cuidados paliativos}

Os médicos entrevistados definiram cuidados paliativos como o cuidado oferecido ao paciente durante o processo de morrer, com o objetivo de melhorar a qualidade de seus últimos momentos. A ideia de cuidados paliativos como uma modalidade de assistência voltada exclusivamente para a fase de final de vida ainda está muito presente no discurso dos entrevistados. Essa ideia de cuidados paliativos como "não há mais nada a fazer", ou seja, cuidados de segunda linha, ainda é predominante na fala dos médicos. Usar a palavra "só" denota aquilo que restou, como se as ações paliativas não fossem prioritárias durante todo o processo de tratamento.

\begin{abstract}
Cuidado paliativo pra mim é um cuidado que você dá no final da vida ou quando a pessoa tem uma doença terminal na qual você não vai mais investir em tratamento terapêutico; e aí você vai dar só conforto de dor, respiratório, para que o paciente tenha um final confortável... Pra você não fazer distanásia, não prolongar a vida da pessoa demais e também não deixar o paciente sem suportar. (E12)
\end{abstract}

Embora seja perceptível um conhecimento básico pelos médicos entrevistados acerca de cuidados paliativos, grande

\begin{tabular}{|c|c|c|c|c|}
\hline \multicolumn{5}{|c|}{$\begin{array}{l}\text { QUADRO } 1 \\
\text { lo dos participantes da pesquisa }\end{array}$} \\
\hline Entrevistado & $\begin{array}{l}\text { Idade } \\
\text { (anos) }\end{array}$ & Sexo & Especialização/Residência & $\begin{array}{l}\text { Tempo de formado(a) } \\
\text { (anos) }\end{array}$ \\
\hline 1 & 38 & M & Urgência e Emergência & 3 \\
\hline 2 & 36 & M & Geriatria & 10 \\
\hline 3 & 27 & M & Nutrologia & 3 \\
\hline 4 & 43 & $\mathrm{~F}$ & Não & 18 \\
\hline 5 & 29 & $\mathrm{~F}$ & Não & 6 \\
\hline 6 & 35 & M & Cirurgia Geral e Cirurgia Vascular & 11 \\
\hline 7 & 26 & M & Não & 1 \\
\hline 8 & 26 & M & Não & 1 \\
\hline 9 & 48 & F & Clínica Médica e Pneumologia & 22 \\
\hline 10 & 67 & M & Clínica Médica e Medicina do Trabalho & 38 \\
\hline 11 & 38 & M & Ginecologia e Obstetrícia & 4 \\
\hline 12 & 25 & $\mathrm{~F}$ & Não & 1 \\
\hline 13 & 33 & F & Dermatologia e Nutrologia & 7 \\
\hline 14 & 62 & $\mathrm{~F}$ & Cardiologia & 36 \\
\hline 15 & 31 & $\mathrm{~F}$ & Não & 4 \\
\hline 16 & 29 & $\mathrm{~F}$ & Não & 3 \\
\hline
\end{tabular}


parte se restringe à qualidade de vida e alívio dos sinais e sintomas físicos, sendo que uma minoria incluiu o trabalho com os familiares e as questões espirituais que envolvem o fim da vida de um paciente, como também foi demonstrado no trabalho de Brugnolli et al. ${ }^{3}$.

Cuidados paliativos, na verdade, é aquele paciente que não tem perspectiva de cura ou de tratamento curativo [...]; já que a gente não pode tratar a doença em si, como curativo, a gente dá um tratamento mais humanizado para o paciente. Um tratamento mais sintomático [...] A gente não quer que o paciente sofra, que ele [paciente] fique sentindo dor. (E2)

No geral, os entrevistados definiram os pacientes oncológicos como os principais numa abordagem de cuidados paliativos.

É um exemplo muito fidedigno... O paciente terminal oncológico. (E1)

Além dos pacientes oncológicos, outros perfis foram elencados pelos entrevistados como pacientes passíveis de abordagem voltada aos cuidados paliativos.

Esse paciente pode ser qualquer um, mas tem que ter o diagnóstico da doença [...] As causas mais comuns são câncer. Mas existem outras: esclerose múltipla, doenças musculares, cardiopatias. (E5)

Esse perfil mais amplo de pacientes passíveis de abordagem paliativa citado por alguns entrevistados vai ao encontro do que diz a literatura. Segundo Queiroz et al. ${ }^{18}$, pessoas com enfermidades crônicas, evolutivas e progressivas, com prognóstico de vida diminuído, são selecionáveis para os cuidados paliativos; além dessas, podem ser inseridos pacientes portadores de neoplasias e outras doenças crônico-degenerativas, como insuficiência renal, insuficiência cardíaca, demências, doença de Parkinson e doença pulmonar obstrutiva crônica $(\mathrm{DPOC})^{1,13}$.

A maioria dos médicos entrevistados referiu-se aos pacientes em cuidados paliativos como pacientes "terminais", evidenciando, uma vez mais, que esse tipo de assistência seria sinônimo de cuidados de final de vida, o que, de fato, não é. Atualmente, entende-se por cuidados paliativos a assistência ao paciente com uma doença potencialmente incurável e sua família ${ }^{5}$, ou seja, cuidados curativos e paliativos não deveriam ser considerados como conceitos excludentes, mas, sim, complementares.
Cuidado paliativo no meu entender é o paciente em fase terminal. (E11)

Queiroz et al. ${ }^{18}$ discutem a questão que envolve o uso da palavra "terminal" por profissionais de saúde. Embora ainda muito usada nos locais de atendimento, o que se questiona é que seu uso traria um rótulo e um estigma responsáveis por um desinvestimento dos profissionais e também da família nesses pacientes. Este cenário é o contrário do desejado, que é a oferta de todo o cuidado técnico e humano necessário, objetivando um final de vida digno.

\section{Formação acadêmica}

As entrevistas demonstraram que o contato dos médicos com os cuidados paliativos durante a formação acadêmica foi mínimo. Nenhum dos entrevistados teve uma disciplina específica voltada para a temática, que, quando trabalhada, foi de forma conjunta com outras disciplinas, por meio de palestras ou do contato com pacientes na vivência prática do curso.

\footnotetext{
A parte de cuidados paliativos foi junto com as outras [...] Junto com a clínica, com o internato rural. Mas, específico, direcionado, não. (E6)
}

Na entrevista dos médicos formados há mais de dez anos, ficou ainda mais evidente a ausência do assunto nas aulas, o que está diretamente relacionado ao fato de os cuidados paliativos ainda não serem reconhecidos como área de atuação do profissional médico na época ${ }^{19}$.

Poucos médicos entrevistados demonstraram maior contato com a área, seja devido a uma especialização, seja por meio de contato em atividades extracurriculares, conforme evidenciado a seguir:

$$
\begin{aligned}
& \text { Eu fiquei muito tempo fazendo estágio no Instituto Oncológico } \\
& \text { de J.F. e aí eu lidava com pacientes fora de possibilidades tera- } \\
& \text { pêuticas [...] eu fui presidente da Liga Acadêmica de Oncologia. } \\
& \text { Aí eu realizei um simpósio sobre medicina paliativa e chamei } \\
& \text { um profissional da área para falar sobre o assunto. Foi a única } \\
& \text { coisa que teve dentro da faculdade com que eu tive contato. (E7) }
\end{aligned}
$$

Os dados corroboram as constatações de Fonseca e Geovanini $^{13}$, que chamam atenção para a pouca abordagem sobre a morte e o morrer durante a formação médica e os esparsos cursos de especialização ou pós-graduação em cuidados paliativos. Com isso, os estudantes pouco desenvolvem as habilidades humanitárias e emocionais necessárias quando se trabalha na área e formam-se como profissionais pouco dispostos 
a refletir e a conversar com o paciente e a família diante da irreversibilidade do quadro clínico. Esse despreparo é resultado de questões sociais e espirituais dos próprios médicos e também do ensino da área da saúde, que prioriza o conhecimento técnico-científico em detrimento de uma abordagem social, emocional e espiritual ${ }^{20}$. E, conforme reforçado por Queiroz et $a l{ }^{18}$, são várias as dimensões de formação necessárias à realização de um bom atendimento a um paciente em final de vida.

Quando questionados se se sentem preparados para atender a um paciente em cuidados paliativos, a maioria dos entrevistados respondeu que não ou não totalmente. Entre os vários motivos, destaca-se a ausência de formação acadêmica para esse cuidado, conforme abordado anteriormente.

Na verdade, totalmente preparado não. Sempre tem um pouco de insegurança, porque isso é uma coisa muito nova. (E2)

Conforme apontado por Rego e Palácios ${ }^{21}$, os cuidados oferecidos a pacientes em final de vida não devem ser de competência exclusiva de médicos especialistas, mas, sim um marco importante na formação de uma equipe de Saúde da Família. Sabe-se que é de competência da Saúde Pública contribuir para a estruturação de serviços de saúde que contemplem a questão do cuidado a esses pacientes. Isto pode ser realizado por meio da implementação de políticas específicas para formação e atualização não só dos médicos, como também de enfermeiros e outros profissionais da Equipe de Saúde da Família que têm contato com pacientes em cuidados paliativos. É importante ressaltar que essa discussão não deve se restringir apenas ao campo técnico, mas abordar aspectos humanísticos, imprescindíveis ao fornecimento de um cuidado integral.

\section{Experiências de atendimento}

Nas entrevistas, os médicos relataram já terem atendido pacientes em cuidados paliativos, embora a maioria tenha afirmado que a demanda seja pouca. A questão que se coloca, diante disso, é: será que realmente a demanda é baixa ou a equipe não sabe identificar o paciente que necessita de cuidados paliativos? Talvez a deficiência esteja no conhecimento necessário à identificação desse tipo de paciente e não na demanda propriamente dita.

Olha, uma demanda [de pacientes em cuidados paliativos] grande não! Mas a gente tem uns casos [...] aqui na área deve ter uns seis ou sete. Não é um número alto, mas tem. (E6)

Estudos demonstraram que os serviços de saúde têm dificuldade em identificar os pacientes em final de vida e que ne- cessitam de uma abordagem voltada aos cuidados paliativos. Quando isso é feito, faltam informações sobre a doença que os acomete, suas condições sociais e possíveis dependências no dia a dia ${ }^{22,23}$.

Entre os motivos do pequeno atendimento desses pacientes na Estratégia Saúde da Família, foi levantada uma condição socioeconômica melhor, que permitia o acompanhamento com médicos especialistas, e também a falta de vínculo com a Unidade de Saúde, o que faz com que os pacientes nem busquem esse atendimento nas Unidades Estratégia Saúde da Família.

Essa área do PSF aqui, eu não estou aqui há muito tempo, mas eu acho que é uma área com uma condição socioeconômica e cultural melhor. Então, você vê que muitos dos pacientes [...] já fazem um tratamento com outros especialistas: geriatras, neurologistas. (E6)

Apesar de o número de pacientes ser reduzido, os relatos de atendimento desses pacientes pelos médicos das Unidades Estratégia Saúde da Família demonstraram as várias possibilidades de atuação existentes quando se pensa em melhorar a qualidade de vida e de morte dos pacientes em cuidados paliativos.

O último foi um paciente oncológico mesmo. Um paciente até novo... 50 e poucos anos [...] já estava em um quadro avançado, já tinha metástase [...] aí chegou uma hora em que não tinha mais o que fazer. A gente falou: pode deixar em casa mesmo, a gente passou um medicamento derivado da morfina. Porque ele [paciente] ia mais para o hospital para tomar analgésico. A gente quis até dar uma ajuda para ele [paciente] ficar mais próximo da família. (E2)

A literatura ressalta as várias necessidades dos pacientes em final de vida, as quais envolvem receber cuidados para o controle da dor, dispneia, constipação intestinal e náuseas, por exemplo, além de receber suporte social, emocional e espiritual, ter acesso a informações, participar das discussões acerca da sua vida e condição de saúde e, por fim, buscar o significado da vida ${ }^{22,24}$.

Sabe-se que o cuidado integral e a atenção voltada ao contexto pessoal e familiar são objetivos do cuidado oferecido tanto na Atenção Primária em Saúde quanto pelos Cuidados Paliativos. Já foi demonstrado que dessa forma há uma influência positiva na qualidade de vida e também de morte dos pacientes. Por isso, é importante que haja um grande conhecimento da população adscrita pelos profissionais que traba- 
lham nas Unidades Saúde da Família para que, dessa forma, sejam identificados e acompanhados os pacientes em cuidados paliativos ${ }^{8,22}$.

\section{Sentimentos relacionados à morte}

Os entrevistados frequentemente afirmaram que se sentiram impotentes e frustrados frente à possibilidade de morte do paciente. Pensando que a formação acadêmica prepara o médico para salvar vidas, ao se deparar com a impossibilidade de cura ou com a possibilidade real de morte, o profissional tende a se sentir frustrado e incapaz:

E foi muito frustrante. Eu tentei ajudar no que eu podia, mas a gente fica assim com as mãos um pouco atadas. (E4)

É o lado frágil da medicina. É você saber lidar com as decepções da vida, em relação aos pacientes que você não tem condição de fazer muito por eles. Então, é decepção diante da morte e da impotência. (E9)

Para alguns entrevistados, o sentimento de impotência estava atrelado a outras situações que não somente a impossibilidade de mudança do prognóstico do paciente, mas, sim, à ausência de recursos para fornecer o melhor cuidado:

Quando a gente não consegue fazer algo pelo paciente, não por nossa culpa, mas por causa do Estado, a gente fica com um sentimento de impotência, porque você vê aquele paciente sofrendo e não pode fazer nada. (E11)

Figueiredo e Stano ${ }^{14}$ levantam, em seu estudo, diferentes razões pelas quais o médico enfrenta a morte de um paciente com frustração e decepção. Sabe-se que o avanço tecnológico e científico produziu uma corrida na busca da cura das doenças, e cada vez mais o médico é induzido a pensar que a cura das doenças é o único motivo pelo qual vale a pena trabalhar, sendo a morte de um paciente o fracasso da sua atuação. $\mathrm{O}$ médico da sociedade atual é competente em lutar contra a doença, porém nem sempre em cuidar do ser humano doente, tomando para si uma postura de negação da morte, comum a toda a sociedade ocidental, no geral. Pouco se estuda sobre os aspectos emocionais e espirituais relacionados ao adoecimento e muito menos sobre como abordar o paciente que não tem mais possibilidades terapêuticas de cura ${ }^{20,25}$.

Para alguns entrevistados, houve uma mudança na forma de encarar a perda e de lidar com o paciente em final de vida. Se antes a conduta era muito intervencionista e descrente em relação aos Cuidados Paliativos, hoje se busca oferecer um final de vida digno. Essa mudança de concepção, segundo os entrevistados, está atrelada principalmente à experiência e à aquisição da consciência de que em muitos casos a morte é inevitável:

\begin{abstract}
Logo depois de formada, eu tive muita dificuldade para lidar com esse tipo de paciente. Eu não me interessava mesmo por eles [pacientes em final de vida], pensava: "ah, não tem mais nada que eu possa fazer" [...] Eu era bem intervencionista. Era até um orgulho resolver no momento... O paciente estava lá, era meu, e tudo que eu podia fazer eu fazia. Mas aí eu comecei a trabalhar em PSF, muito por causa dessa humanidade que estava perdendo. E aqui a gente tem uma outra visão sobre tudo isso. A gente é mais próxima do paciente, da família. E aí eu comecei a pegar gosto em acompanhar esses pacientes.

$(\mathrm{E} 13)$
\end{abstract}

Ainda segundo alguns relatos, logo após a formação como médico, a tendência era de um grande sofrimento pessoal quando se deparavam com a perda de um paciente. Fonseca e Geovanini ${ }^{13}$ pontuam que a dificuldade ao lidar com a perda de pacientes pode levar a consequências emocionais para a vida do profissional e sérias dificuldades no trabalho, como foi relatado por um dos entrevistados:

Eu não conseguia aceitar não [...] foi bem difícil. O óbito me deixava mal. Aí eu vi o quanto estava me atrapalhando e resolvi fazer terapia, o que me ajudou muito. Eu consegui achar uma forma de lidar melhor com isso, e hoje eu estou bem melhor. (E13)

Constatou-se também que a experiência contribuiu para que hoje os profissionais consigam atender os pacientes em final de vida com humanidade, mas sem se envolver a ponto de prejudicar seu trabalho e vida pessoal. A morte passou a ser vista com mais naturalidade em situações em que não há possibilidade terapêutica de cura. A questão não é demonstrar insensibilidade perante o sofrimento do paciente e da família, mas, sim, dosar o envolvimento de forma que se consiga estabelecer uma relação médico-paciente dentro de limites "confortáveis":

[...] quando a gente vai trabalhando no dia a dia, aí vai tolerando essas coisas [...] E aí a gente vai ficando mais acostumada [...] ser médico leva a isso. Acostumar-se a essas situações [...] Mas a gente sempre sofre com essas coisas, de forma geral. (E5)

As falas dos entrevistados reforçam a necessidade de maior abordagem dos cuidados paliativos durante a formação acadêmica. Como não houve esse trabalho durante a formação 
médica, foi somente com as dificuldades do dia a dia de atendimentos e a aquisição de experiência que os profissionais entrevistados se sentiram mais confortáveis no acompanhamento dos pacientes em fim de vida. Sabe-se que a experiência é de extrema importância e realmente só é adquirida com o trabalho. Porém, uma parte do sofrimento pessoal e moral desses médicos poderia ser diminuída se temas relacionados à morte, ao morrer e à perda de pacientes fossem mais discutidos nas escolas médicas ${ }^{13,26,27}$

\section{Limitações dos profissionais e do sistema de saúde}

Quando questionados sobre as dificuldades que enfrentam ao atender pacientes fora de possibilidades terapêuticas de cura, os entrevistados citaram situações que impedem um cuidado integral ao paciente, sendo que todas podem ser divididas em duas categorias: a limitação pessoal e as limitações do sistema de saúde.

Quanto às limitações pessoais, uma situação apontada foi a dificuldade na comunicação com a família e com o paciente, especialmente nas questões que envolviam a doença e a morte. Talvez essa dificuldade para estabelecer uma comunicação adequada com o paciente e a família não ocorra exclusivamente nas situações de final de vida, mas provavelmente é potencializada pela maior carga emocional que se apresenta quando se trata de lidar com a possibilidade de morte do paciente:

Então você imagina dar uma notícia dessa para uma filha de um pai ou de uma mãe? Aquela tristeza dela te consome. (E1)

Nesse contexto, Geovanini e Braz ${ }^{28}$ ressaltam que, embora possam ocorrer em qualquer atividade relacional, as dificuldades de comunicação tendem a ser maiores nas situações que envolvem doença e morte, devido às limitações de ordem pessoal do profissional, pois, quando se depara com a morte de um paciente, pensa na própria finitude; e também em virtude do despreparo profissional para realizar essa tarefa. Assim como a morte não é discutida nas escolas médicas, a comunicação de más notícias também não é tema nas matrizes curriculares. Dessa forma, os profissionais se sentem incapacitados para essa tarefa e mostram preocupação em como a má notícia afetará o paciente; preocupação de causar dor e sofrimento ao indivíduo; culpabilização por parte do paciente em seu diagnóstico; incertezas sobre a terapêutica e as várias reações emocionais causadas pela informação da possibilidade de morte ${ }^{29,30}$.

Quanto às limitações relacionadas ao sistema, a mais relatada foi a dificuldade de conciliar a grande demanda de atendimento das Unidades Estratégia Saúde da Família com o acompanhamento dos pacientes:
Assim... a gente tem uma demanda muito grande aqui dentro do PSF. E tem uma demanda grande de fora para fazer visita domiciliar. Só que nem sempre a gente consegue conciliar esses dois... [...] É mais essa questão de que às vezes eu queria ser dois. (E7)

Essa limitação também foi descrita por Queiroz et al. ${ }^{18}$, que afirmam que a grande e variada demanda na Atenção Primária pode impedir o fornecimento de uma assistência adequada ao paciente em final de vida e aos seus cuidadores. É interessante notar que a maioria dos pacientes em Cuidados Paliativos encontra-se restrita ao lar devido a incapacidades próprias da doença, acarretando a necessidade de atendimento domiciliar. Daí a dificuldade de conciliar esse tipo de atendimento numa agenda muitas vezes sobrecarregada.

Outra dificuldade relatada foi o estabelecimento de vínculo com os pacientes, visto que os médicos são sempre trocados de Unidade de Saúde dentro da Estratégia de Saúde da Família no referido município:

A rotatividade no PSF é muito grande. Você está acompanhando um paciente, aí muda de PSF e nem fica sabendo mais o que aconteceu [...] infelizmente, você perde muito o vinculo com o paciente. (E4)

É sabido que a Atenção Primária em Saúde se orienta pelos conceitos de longitudinalidade, integralidade, humanização, coordenação do cuidado, vínculo e continuidade ${ }^{31}$. Todos esses conceitos também são essenciais no atendimento ao paciente em cuidados paliativos, que tem como objetivo apoiar a família a cumprir sua função cuidadora, vivenciando de forma menos traumática a perda e o luto. Quando se quebra o vínculo, esse acompanhamento é mais difícil de ser realizado, influenciando a forma como a perda do paciente é encarada $^{32}$.

Outro entrave presente na fala dos entrevistados foi a falta de insumos para um bom atendimento aos pacientes. Foi relatada demora na realização de exames e na marcação de consultas com médicos especialistas. Além disso, foram apontadas dificuldades com o meio de transporte da Prefeitura para as visitas domiciliares e também a ausência de medicações para dor, sendo que muitos pacientes não têm condições econômicas para a aquisição de tais medicamentos:

Nós [Equipe de Saúde da Família] temos uma limitação de carro pra fazer visita. Porque é um carro só para todos os PSF. (E9) 
E existem as dificuldades da Saúde Pública. Às vezes, um exame que demora muito, uma consulta que demora muito. (E14)

Outro problema é a questão de medicação para dor. A maioria dos medicamentos para dor não está disponível no SUS. E esses pacientes não têm condição para ficar comprando remédio caro. (E13)

A escassez de recursos é uma realidade não apenas para os atendimentos em Cuidados Paliativos, mas também em diversas outras áreas da Saúde Pública. Frossardi ${ }^{33}$ reforça que os cuidados paliativos expressam a realidade da saúde brasileira: ainda sem todos os investimentos necessários. Apesar de iniciativas que buscam a introdução da área na Atenção Primária por meio, por exemplo, do Programa Melhor em Casa, muitos ainda são os problemas de ordem prática que impedem a prestação de um adequado serviço de cuidados paliativos, como relatado pelos entrevistados. A resolução desse problema envolve uma série de fatores, mas é necessário que ocorra maior difusão do assunto na sociedade em geral e um real envolvimento das entidades públicas, com programas voltados à melhoria dos serviços de saúde para a população, entre os quais se incluem os cuidados a pacientes em final de vida.

\section{CONSIDERAÇÕES FINAIS}

Os profissionais que trabalham na Estratégia Saúde da Família acompanham seus usuários nas várias condições e situações que levam um indivíduo a buscar atendimento em saúde. A morte, assim como o nascimento, demanda esse acompanhamento e cuidado. Nenhum outro serviço de saúde pode oferecer essa assistência com tanta propriedade como a Atenção Primária em Saúde, justamente porque se baseia no cuidado humanizado e longitudinal e no estabelecimento de vínculo com os pacientes e famílias.

Apesar disso, o papel da ESF nos cuidados de final de vida ainda é muito restrito, limitando-se a procedimentos de retaguarda à família e alguns procedimentos para o bem-estar do paciente. Essa restrição deve-se, em muito, à ausência da abordagem dos cuidados paliativos na formação acadêmica de médicos, enfermeiros e outros profissionais de saúde, que não sabem exatamente o que deve ser feito quando possuem demandas por esse tipo de cuidado.

Embora as políticas voltadas aos cuidados paliativos ainda sejam incipientes, a Plenária do Conselho Federal de Medicina aprovou por aclamação o documento norteador da política de cuidados paliativos para o SUS. Diversos conselhei- ros federais se manifestaram, parabenizando a qualidade do documento e ressaltando sua importância para o nosso país. Esperam-se os trâmites finais rumo à publicação da Portaria de Cuidados Paliativos para o SUS.

A recomendação da Organização Mundial da Saúde de ampliar o acesso de pacientes aos serviços de cuidados paliativos torna necessária uma discussão maior sobre a área durante a formação médica, além do desenvolvimento de políticas públicas que incluam as ações em cuidados paliativos na Atenção Primária e o treinamento dos profissionais de saúde já formados, para que seja possível proporcionar melhor qualidade de vida e de morte.

\section{REFERÊNCIAS}

1. Academia Nacional de Cuidados Paliativos (ANCP). Manual de cuidados paliativos. Rio de Janeiro: Diagraphic, 2009.

2. Souza HL, Zoboli ELCP, Paz CRP, Schveitzer MC, Hohl KG, Pessalacia JDR.Cuidados paliativos na atenção primária à saúde: considerações éticas. Rev Bioét. (Impr.) 2015; 23(2) 349-359.

3. Brugugnolli ID, Gonsaga RA, Silva EM. Ética e cuidados paliativos: o que os médicos sabem sobre o assunto? Rev Bioét 2013; 21(3) 477-485.

4. Afonso SBC, Minayo MCS. Uma releitura da obra de Elisabeth Kubler-Ross. Ciênc. Saúde Colet. 2013;18(9) 27292732.

5. World Health Organization (WHO). Definition of palliative care. Disponível em: http://www.who.int/cancer/ palliative/definition/en/. Acesso 01 Novembro 2017.

6. Costa AP, Poles K, Silva AE. Formação em cuidados paliativos: experiência de alunos de medicina e enfermagem. Interface 2016; 20(59) 1041-1052.

7. Conselho Federal de Medicina. Resolução CFM nº 1.973/2011. Dispõe sobre a nova redação do Anexo II da Resolução CFM nº 1.845/08, que celebra o convênio de reconhecimento de especialidades médicas firmado entre o Conselho Federal de Medicina (CFM), a Associação Médica Brasileira (AMB) e a Comissão Nacional de Residência Médica (CNRM). Diário Oficial da União. Brasília, 01 ago. 2011; Seção 1, p. 144-147.

8. Silva MLSR. O papel do profissional da Atenção Primária à Saúde em cuidados paliativos. Rev Bras Med Fam Comunidade 2014; 9(30) 45-53.

9. Brasil. Mistério da Saúde. Portaria GM/MS no 19, de 03 de janeiro de 2002. Institui no âmbito do Sistema Único de Saúde - SUS, o programa nacional de assistência à dor e cuidados paliativos. Diário Oficial da União. Brasí- 
lia, 03 jan. 2002. Disponível em: http://bvsms.saude.gov. br/bvs/saudelegis/gm/2002/prt0019_03_01_2002.html. Acesso em 21 de julho de 2016.

10. Brasil. Lei $\mathrm{n}^{\mathrm{o}}$ 10.424, de 15 de abril de 2002. Acrescenta capítulo e artigo à Lei no 8.080, de 19 de setembro de 1990, que dispõe sobre as condições para a promoção, proteção e recuperação da saúde, a organização e o funcionamento de serviços correspondentes e dá outras providências, regulamentando a assistência domiciliar no Sistema Único de Saúde. Diário Oficial da União. Brasília, 16 abr. 2002; Seção 1, p. 1. Disponível em: http://www.planalto.gov.br/ccivil_03/ leis/2002/110424.htm. Acesso em 08 de julho de 2016.

11. Brasil. Ministério da Saúde. Secretaria de Atenção à Saúde. Departamento de Atenção Básica. Caderno de atenção domiciliar / Ministério da Saúde, Secretaria de Atenção à Saúde, Departamento de Atenção Básica. - Brasília: Ministério da Saúde, 2012.

12. Baliza mf, Bousso RS, Spineli VMCD, Silva L, Poles K. Cuidados paliativos no domicílio: percepção de enfermeiras da Estratégia Saúde da Família. Acta Paul Enferm 2012; 25(Número especial 2) 13-18.

13. Fonseca A, Geovanini F. Cuidados paliativos na formação do profissional da área de saúde. Rev Bras Edu Med 2013; 37(1) 120-125.

14. Figueiredo MGMCA, Stano RCMT. O estudo da morte e dos cuidados paliativos: uma experiência didática no currículo de medicina. Rev Bras Edu Med 2013; 37(2) 298-307.

15. Polit DF, Hungler BP. Pesquisa e análise qualitativa: fundamentos de pesquisa em enfermagem. 3 ed. Porto Alegre: Artes Médicas, 1995.

16. Bardin L. Análise de conteúdo. Lisboa: Edições 70, 2006.

17. Brasil. Ministério da Saúde. Conselho Nacional de Saúde. Resolução no 466, de 12 de dezembro de 2012. Regulamenta a realização de pesquisas envolvendo seres humanos no Brasil. Diário Oficial da União. Brasília, 13 jun. 2013. Disponível em http://bvsms.saude.gov.br/bvs/saudelegis/cns/2013/res0466_12_12_2012.html. Acesso em 25 de agosto de 2016.

18. Queiroz AHAB, Pontes RJS, Souza AMA, Rodrigues TB. Percepção de familiares e profissionais sobre os cuidados no final de vida no âmbito da atenção primária à saúde. Ciênc. Saúde Colet. 2013; 18(9) 2615-2623.

19. Hermes HR, Lamarca ICA. Cuidados paliativos: uma abordagem a partir das categorias profissionais de saúde. Ciênc. Saúde Colet. 2013; 19(9) 2577-2588.

20. Kovács MJ. Educação para a morte: temas e reflexões. Editora Fapesp, 2003.
21. Rego S, Palácios M. A finitude humana e a saúde pública. Cad. Saúde Pública 2006; 22(8) 1755-1760.

22. Marcucci FCI, Perilla AB, Brun MM, Cabrera MAS. Identificação de pacientes com indicação de Cuidados Paliativos na Estratégia Saúde da Família: estudo exploratório. Cad. Saúde Colet. 2016; 24 (2) 145-152.

23. Valente $\mathrm{SH}$, Teixeira MB. Estudo fenomenológico sobre a visita domiciliária do enfermeiro à família no processo de terminalidade. Rev Esc Enferm USP 2009; 43(3) 655-661.

24. Menezes RA. Em busca da boa morte: antropologia dos cuidados paliativos. Rio de Janeiro: Garamond/Fiocruz; 2004.

25. Moritz, RD. Os profissionais de saúde diante da morte e do morrer. Bioética 2005; 13(2) 51-63.

26. Lustosa AM, Dutra F, Moreira MADM, Evangelista CB, Duarte MCS, Zaccara AAL et al. Cuidados Paliativos: discurso de médicos residentes. Rev Med Minas Gerais 2015; 25(3) 369-374.

27. De Moraes SAF, Kairalla MC. Avaliação dos conhecimentos dos acadêmicos do curso de medicina sobre os cuidados paliativos em pacientes terminais. Einstein (São Paulo) 2010; 8(2) 162-167.

28. Geovanini F, Braz M. Conflitos éticos na comunicação de más notícias em oncologia. Rev Bioét 2015; 21(3) 455-462.

29. Floriani CA, Schramm FR. Desafios morais e operacionais da inclusão dos cuidados paliativos na rede de atenção básica. Cad Saúde Pública 2007; 23(9) 2072-2080.

30. Sombra Neto LL, Silva VLL, Lima CDC, Moura HTM, Gonçalves ALM, Pires APB et al. Habilidade de Comunicação da Má Notícia: o Estudante de Medicina está preparado? Rev Bras Edu Med 2017; 41(2) 260-268.

31. Brasil. Ministério da Saúde. Secretaria de Atenção à Saúde. Departamento de Atenção Básica. Política Nacional de Atenção Básica / Ministério da Saúde. Secretaria de Atenção à Saúde. Departamento de Atenção Básica. - Brasília: Ministério da Saúde, 2012

32. Hennemann-Krause L, Freitas LA, Daflon PMN. Cuidados paliativos e medicina de família e comunidade: conceitos e interseções. Revista HUPE 2016; 15(3) 286-293.

33. Frossardi A. Os cuidados paliativos como política pública: notas introdutórias. Cad EBAPE 2016; 14(n. esp.) 640-655. 


\section{CONTRIBUIÇÃO DOS AUTORES}

Júlia Rezende Ribeiro: coleta e análise dos dados, idealização e elaboração do artigo. Kátia Poles: orientação da análise dos dados, idealização e elaboração do artigo.

\section{CONFLITO DE INTERESSES}

Não há conflito de interesses.

\section{ENDEREÇO PARA CORRESPONDÊNCIA}

Rua Vinte de Novembro, 320

Jardim Fabiana - Lavras

CEP 37200-000 - MG

E-mail: katia.poles@ufla.br. 